\title{
Neuroimaging in Animal Seizure Models with ${ }^{18}$ FDG-PET
}

\author{
Martine M. Mirrione ${ }^{1,2,3}$ and Stella E. Tsirka ${ }^{1}$ \\ ${ }^{1}$ Department of Pharmacological Sciences, Molecular and Cellular Pharmacology, Stony Brook University, Stony Brook, \\ NY 11794-8651, USA \\ ${ }^{2}$ Medical Department, Brookhaven National Laboratory, Upton, NY 11973, USA \\ ${ }^{3}$ Cold Spring Harbor Laboratory, Cold Spring Harbor, NY 11724, USA
}

Correspondence should be addressed to Martine M. Mirrione, mmirrion@cshl.edu

and Stella E. Tsirka, stella@pharm.stonybrook.edu

Received 13 October 2010; Revised 24 February 2011; Accepted 31 March 2011

Academic Editor: Helen E. Scharfman

Copyright (C) 2011 M. M. Mirrione and S. E. Tsirka. This is an open access article distributed under the Creative Commons Attribution License, which permits unrestricted use, distribution, and reproduction in any medium, provided the original work is properly cited.

\begin{abstract}
Small animal neuroimaging has become increasingly available to researchers, expanding the breadth of questions studied with these methods. Applying these noninvasive techniques to the open questions underlying epileptogenesis is no exception. A major advantage of small animal neuroimaging is its translational appeal. Studies can be well controlled and manipulated, examining the living brain in the animal before, during, and after the disease onset or disease treatment. The results can also be compared to data collected on human patients. Over the past decade, we and others have explored metabolic patterns in animal models of epilepsy to gain insight into the circuitry underlying development of the disease. In this paper, we provide technical details on how metabolic imaging that uses 2-deoxy-2 $\left[{ }^{18} \mathrm{~F}\right]$ fluoro-D-glucose $\left({ }^{18} \mathrm{FDG}\right)$ and positron emission tomography (PET) is performed and explain the strengths and limitations of these studies. We will also highlight recent advances toward understanding epileptogenesis through small animal imaging.
\end{abstract}

\section{Introduction}

There is a strong foundation for using neuroimaging techniques such as PET to probe circuitry underlying symptoms of patients with epilepsy. In this paper, we will highlight basic research in this area, focusing our discussion on studies conducted using imaging on animal models.

What makes using neuroimaging techniques so advantageous? First, these methods can be harnessed to dissect circuitry differences between various classifications of epilepsy. There are currently over 40 classified types of epilepsy, including generalized, focal, continuous, and reflex seizures according to the International League against Epilepsy [1]. There are also over 27 identified epilepsy syndromes classified by a myriad of seizure types, symptoms, pathology, and etiology. PET can potentially be used in the evaluation and diagnosis of differing patient populations.

Second, for a given radiotracer, brain scans from patients can be almost directly compared to small animal neuroimaging results, which can validate existing models. Even though seizure symptoms and classifications are so diverse and animal models only partially exemplify aspects of the human condition, a rich basic science literature exists that has significantly advanced our understanding of circuitry changes underlying seizures using techniques such as electrophysiology and microscopy to measure cellular changes. PET has contributed and will continue to contribute to our understanding in these areas through its major advantage of conducting whole brain analyses. Prior research has validated the use of 2-deoxy-2 $\left[{ }^{18} \mathrm{~F}\right]$ fluoro-D-glucose ( $\left.{ }^{18} \mathrm{FDG}\right)$, a radiotracer which measures tissue metabolic activity, toward this goal. Therefore as a foundation, we and others have explored circuitry in the epileptic animal model using ${ }^{18}$ FDG-PET imaging methods [2-8]. Although the limited spatial resolution of PET does not allow for the evaluation of changes at the cellular level, this disadvantage is balanced by the ability to look at overall changes throughout brain regions related to seizure symptoms. Furthermore, newer ligand-specific radiotracers make possible a more precise examination of neurotransmitter systems [9-12]. 
Third, neuroimaging is considered noninvasive and thus several scans can be given to the same patient over the course of the disease, or the same animal over the time course of an experiment. Therefore, the progression from naïve to acute and then to chronic seizures can truly be studied and perhaps more importantly, new treatments can be evaluated in animal subjects over time. A patient (or animal model) that has more than one seizure over time is considered to be epileptic, and the progression of epilepsy from an initial single seizure to spontaneous recurrent seizures (SRS) is called epileptogenesis. Continuing research efforts to understand the mechanisms of epileptogenesis are required to identify new molecular targets which may help control refractory seizures. Small animal neuroimaging techniques are useful in this endeavor, offering the advantage that they allow investigators to examine the living brain before the disease is induced and over the time course of symptoms and even therapies.

In the next few sections, a technical review of ${ }^{18}$ FDG-PET is given, followed by a discussion of what we have learned and can still learn by studying human epilepsy in animal models with these strategies.

\section{Technical Aspects of ${ }^{18}$ FDG-PET}

Three decades have passed since imaging was introduced to diagnostic medicine. Techniques such as X-ray computed tomography (CT) and magnetic resonance imaging (MRI) have allowed practitioners to evaluate structures noninvasively within patients. The evolution of positron emission tomography (PET) radiotracers has provided the opportunity to evaluate function within specific tissues or organs. One of the most widely used tracers in PET is ${ }^{18} \mathrm{FDG}$, which measures glucose metabolism and has been used extensively in diagnosis of cancer and epilepsy. More recently, ${ }^{18} \mathrm{FDG}-$ PET has shown promise in the analysis of other disorders as well, including Alzheimer's disease and depression [13, 14]. Epileptic patients show interesting metabolic signatures both in-between (interictal) and during (ictal) seizures, which have helped in diagnosis and presurgical evaluation $[15,16]$.

Neuroimaging techniques have advantages and limitations. In the following paragraphs, we will discuss these characteristics along with technical aspects of conducting a PET scan for those unfamiliar with this imaging method.

${ }^{18} \mathrm{FDG}$ radiopharmaceutical production begins with conversion of ${ }^{18} \mathrm{O}$ enriched water to the isotope ${ }^{18} \mathrm{~F}$ at a cyclotron, followed by incorporation of the fluoride isotope onto the deoxyglucose molecule to make ${ }^{18} \mathrm{FDG}$ (Figure 1(a)) [17]. After pyrogen testing, a trace amount of ${ }^{18} \mathrm{FDG}$ is administered to a subject, where it accumulates in metabolically active tissues or organs (Figure 1(b)). As each molecule of ${ }^{18} \mathrm{FDG}$ undergoes beta decay, a neutron and a positron are released. When the positron collides with a nearby electron (the range of which provides an inherent resolution limitation), an annihilation event occurs producing two $511 \mathrm{keV}$ gamma rays released nearly $180^{\circ}$ from each other. The noncollinearity of the gamma rays (or slight deviation from exactly $180^{\circ}$ ) provides a second physical limitation to resolution. These are detected as coincidence events by two opposite lutetium oxyorthosilicate (LSO) crystals (or other detector material) contained in the cylinder surrounding the subject in the PET camera bed portal (Figure 1(c)). Coincidence events can also occur at random, and actual events can be missed due to scatter effects introducing artifact in the data sets. Corrections for scatter effects [18], attenuation, dead time, and detector sensitivity can potentially reduce noise.

Software programs used in conjunction with the PET camera record coincidence events and reconstruct where the decays were most likely to have occurred within the field of view. The most common reconstruction algorithm is Filtered Back Projection (FBP), which has the advantage of economical computing resources, but is restricted by noise artifacts and relatively lower spatial resolution. Expectation maximization algorithms for reconstruction of PET data were first employed over 2 decades ago [19], however at the time their demanding computing resources had restricted their clinical availability and acceptance. Many groups have developed variations on the approach reducing computational burden, decreasing time to convergence, thus allowing the algorithms to become increasingly practical for routine use [20]. Such iterative methods include orderedsubsets expectation maximization (OSEM) [21], maximum likelihood expectation maximization (MLEM) [22], and maximum a posteriori (MAP) [23]. The actual resolution depends on several factors, including the physics of the annihilation event (discussed above) and specifications of the camera used to collect the data (such as scintillation crystal performance and electronics). Data can be improved with the reconstruction methods employed but still with limitations. Typically, small animal PET scanners with a reduced ring diameter and portal size approach a spatial resolution range of $1-3 \mathrm{~mm}$ FWHM [24, 25], while larger human scanners can range from 4-7 mm FWHM [26].

The pharmacokinetics of ${ }^{18} \mathrm{FDG}$ have been well studied and involve a four compartment model [27]. Normally as glucose from the circulation enters a cell via a glucose transporter (K1), it is converted into glucose-6-phosphate by the action of hexokinase during the first step of glycolysis (K3). Subsequently, glucose phosphate isomerase (GPI) converts this metabolite into fructose-6-phosphate. However, due to the substitution of a fluorine atom, the intracellular conversion of ${ }^{18} \mathrm{FDG}$ by the enzyme hexokinase into FDG-6-phosphate yields an unsuitable substrate for further metabolism by GPI. This simplifies interpretation of results, as there are no further radioactive metabolites to complicate the data or the determination of reaction rate. Furthermore, the rate of efflux (K4) of FDG-6-phospate is very slow, trapping the radioactive molecule in the metabolically active cell. The slow efflux coupled with a half life of 109.8 minutes makes ${ }^{18} \mathrm{FDG}$ well suited for determining metabolic rates of glucose in tissue. It also makes it ideal for studies aimed at evaluating metabolic changes from baseline concurrent with a task or behavior, including seizures. For example, increased neuronal activity during and immediately following a seizure episode would result in metabolic changes in the brain and can be visualized with ${ }^{18} \mathrm{FDG}$. One caveat with ${ }^{18} \mathrm{FDG}$ is that the Harderian glands typically show a high degree of tracer 


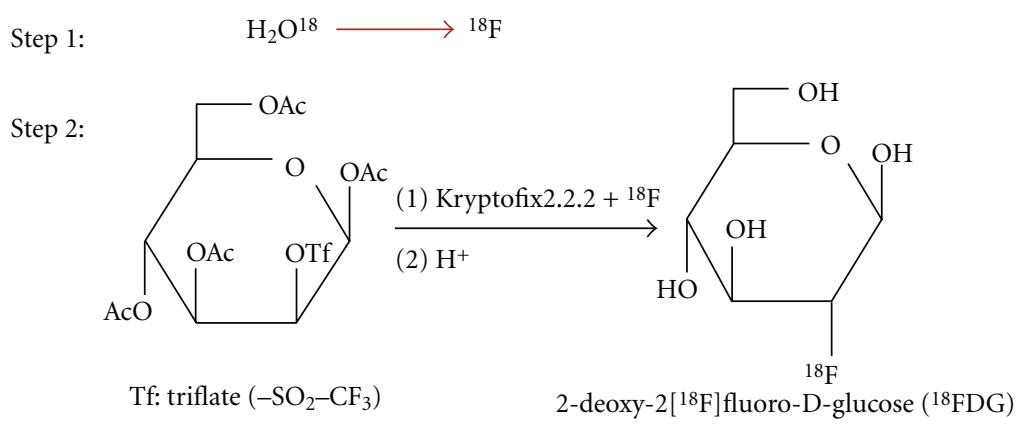

Glucose starting material

(a)

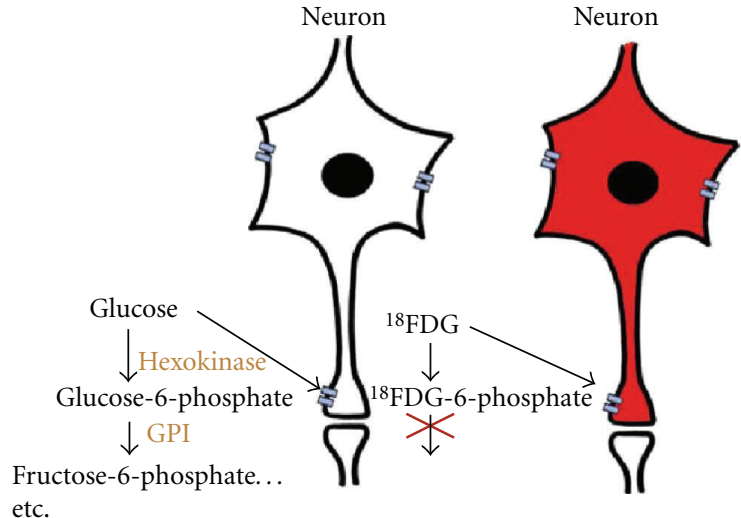

(b)

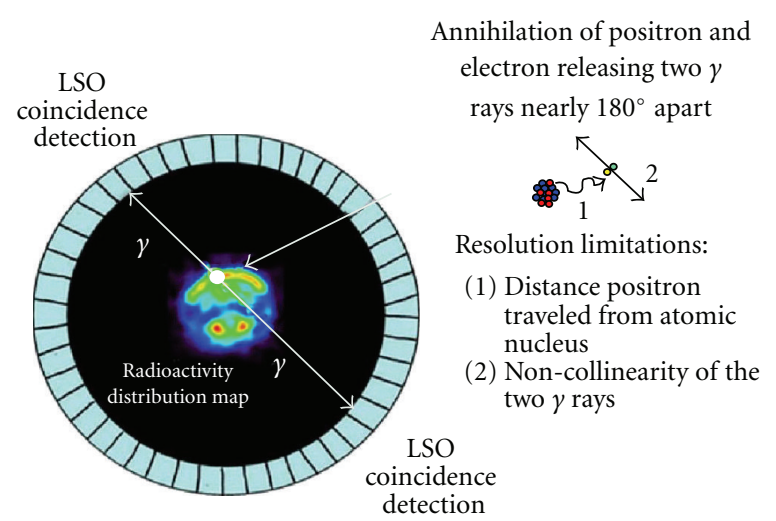

(c)

FIGURE 1: Schematic of ${ }^{18}$ FDG-PET imaging principles. (a) ${ }^{18}$ FDG production step 1: ${ }^{18} \mathrm{O}$ enriched water (95\%) is bombarded with protons in a cyclotron creating 18 -flouride $\left({ }^{18} \mathrm{~F}\right)$ which is purified on a resin column and rinsed with potassium carbonate. Step $2:{ }^{18} \mathrm{~F}$ is added with Kryptofix2.2.2 to the precursor glucose starting material and through a nucleophilic substitution, subsequent purification and removal of protecting groups, ${ }^{18} \mathrm{FDG}$ is synthesized. (b) The radiotracer accumulates in tissue where it is transported into metabolically active cells, metabolized by hexokinase, and trapped as FDG-6-phosphate, as it is not a good substrate for further metabolism by glucose phosphate isomerase (GPI). (c) Representation of a mouse in the field of view of a small animal PET camera. When the ${ }^{18} \mathrm{~F}$ on a molecule of ${ }^{18} \mathrm{FDG}$ undergoes beta decay, a proton turns into a neutron expelling a positron and neutrino from the atomic nucleus. The positron travels up to a few millimeters before it collides with an electron (inset). The energy of this annihilation expels $2511 \mathrm{keV}$ gamma rays $(\gamma)$ approximately $180^{\circ}$ apart from each other. These $\gamma$ rays are detected simultaneously by a ring of LSO crystals that make up the PET camera.

uptake and can interfere with measurements made in the olfactory bulb and frontal cortex. Thus despite the limited resolution and the need for injecting a radioactive substance into the subject, the major advantage of PET is the ability to study function of the living brain over time.

\section{Clinical Diagnosis and Neuroimaging Research}

Early on, it was discovered that epileptic patients evaluated with ${ }^{18}$ FDG showed abnormalities in the form of hypometabolic areas in the limbic system depending on the progression of the disease. Clinicians originally hoped to use these scans to identify the region of seizure focus leading surgical resection of the aberrant region. However, it was found that the hypometabolic regions seemed to extend beyond that of the seizure focus, making it difficult to identify the exact margins. Interestingly, studies had shown that during the ictal phase, when ${ }^{18} \mathrm{FDG}$ is administered very close to the onset of spontaneous seizures, there is an expected increase in metabolic activity in the seizure focus
[28]. This of course would be helpful to a physician's strategy to extract the epileptic zone in medically intractable patients and led to development of ictal perfusion SPECT imaging with $99 \mathrm{mTc}$-ethyl cysteinate dimer (ECD) or $99 \mathrm{mTc}$ hexamthylpropyleneamine oxime (HMPAO) [16]. The tracer is injected into the patient during the seizure and, due to its pharmacokinetics, provides a snapshot of the metabolic activity in the brain that can be viewed later (e.g., 30$60 \mathrm{~min}$ for ${ }^{18} \mathrm{FDG}$ ). However, there are technical limitations to this, including coordinating the patients seizure onset with injection and that not all medical centers are equipped for this type of study [29].

\section{Basic Metabolic Neuroimaging Research: Evaluating Circuitry in Animal Seizure Models}

Several research groups, including ours, have employed in vivo brain imaging methods to understand changes in neurocircuitry due to acute and chronic seizures, and to 
study the progression of epilepsy in animal models [2, 3, $5,7,11,30-37]$. Furthermore, many excellent reviews have been written regarding the use and impact of imaging animal models of epilepsy $[6,29,38-41]$. This paper will highlight the important findings of recent studies.

Our initial study examined pilocarpine-induced seizures in $\mathrm{C} 57 \mathrm{Bl} / 6$ mice [3] as a foundation for our subsequent studies in genetically modified mice $[2,30]$. We confirmed that brain activity in wild-type mice can be recorded and visualized during seizures with ${ }^{18}$ FDG-PET. Specifically, we found a $33 \%$ increase in metabolic activity in the hippocampus, which correlated with the degree of seizure severity $\left(r^{2}=0.86\right)$ [3]. This result was consistent with a previous study in rats using kainate [4] and indicated that it was possible to identify regions of elevated neuronal activity during seizures. One of the benefits of ${ }^{18}$ FDG-PET, as discussed in the previous section, is that the radiotracer can be injected into an awake animal behaving normally, and become trapped in metabolically active cells. In our protocol, we inject ${ }^{18} \mathrm{FDG} 10 \mathrm{~min}$ after pilocarpine injection to capture brain activity during the peak of the seizure behavioral response. The animals were then anesthetized 4560 min following the ${ }^{18} \mathrm{FDG}$ injection and a static (1 frame) 10-20 min scan was acquired.

In our next study, we developed a more detailed region of interest (ROI) and voxel-based strategy for analyzing these data. We found interesting correlations between seizures and metabolic activity in wild-type mice using Statistical Parametric Mapping (SPM), an image analysis tool to explore changes throughout the brain without predefining regions of interest. Specifically, we found positive correlations with seizure activity in the hippocampus, septum, thalamus, midbrain, olfactory bulb, and cerebellum [2]. These regional metabolic changes may represent the effect of seizure spreading: while the hippocampus, septum, and thalamus are initially required for seizure generation (as they express muscarinic (M1) acetylcholine receptors-the sites of action of pilocarpine), elevated activity is propagated downstream in additional regions likely through glutamatergic receptor mechanisms.

The neurocircuitry of the rat lithium-pilocarpine model has also been explored using neuroimaging methods $[5,7$, 31 . The studies by Goffin et al. and Guo et al., [5, 7] examined changes in glucose metabolism over time using ${ }^{18}$ FDG-PET. Goffin and colleagues compared rats that had received saline or pilocarpine at three time points, baseline, day 3 following pilocarpine injection in the early silent phase of epileptogenesis and at the end of the monitoring period during the chronic phase (day 70). They found that at day 3 , metabolism in most of the brain (except the cerebellum) was decreased with the biggest changes occurring in the hippocampus, entorhinal cortex, and thalamus as compared to baseline and saline treated [5]. Guo and colleagues found similar patterns conducting brain scans at day 2 (early), day 7 (latent), and day 42 (chronic) phases after initial SE induction. This group showed in the early phase, there was a significant decrease in metabolism in the entorhinal cortex [7]. Interestingly, these results paralleled our data, suggesting that hippocampal regions, which exhibit overwhelming activity during seizure induction, undergo significant changes during the latent period and result in regional hypometabolism.

In the chronic period, Goffen et al. found increased activity bilaterally in the cerebellum, pons, and medulla oblongata, along with decreased activity in the left striatum and entorhinal cortex, when comparing the seizure group to controls. Unfortunately, the method was not sensitive enough to detect changes between animals experiencing SRS and not, as there were only 3 and 4 animals in these groups to compare, respectively. This is understandable, however, since it is relatively difficult to obtain sufficient numbers of surviving animals showing SRS in the chronic phase. Therefore, the study conducted by Guo et al. employed more animals to explore chronic effects. They observed that global hypometabolism persisted through the latent phase but recovered in the chronic phase, except for the hippocampus and thalamus, which remained below baseline in animals that had shown SRS. Throughout the experiment cerebellar metabolism was not changed. Interestingly, severe entorhinal cortex hypometabolism in the early phase correlated with epileptogenesis behaviorally such that a shorter latent phase and higher frequency of SRS correlated with the degree of hypometabolism. These data provide further evidence that reduced neuronal activity in the entorhinal cortex in the early phase, and persistent hypometabolism in the hippocampus and thalamus following SRS, may underlie the progression of epileptogeneisis.

Data from our group also suggested an important role for the dorsolateral entorhinal cortex (as well as the amygdaloid nucleus, piriform cortex, and anterior striatum) during the early (silent) period, as these areas were hypometabolic in wild-type mice 7 days after pilocarpine [30]. We also found increased activity bilaterally in the thalamus, superior colliculus, and cerebellum. However, in our study the mice injected did not experience severe seizures during the induction period (average score based on the above described scale was only $1.88 \pm 0.19)$. This finding highlights an important point that studies should address the differences between drug effects (pilocarpine/chemoconvulsant) alone versus the effect of seizure symptoms. It may be that small animal imaging is not sensitive enough to detect changes between mice experiencing SRS and not (due to intersubject variability and low resolution), however, further studies with larger groups of animals suitable for statistical analysis, either with region of interest (ROI) or whole brain voxel-based (Statistical Parametric Mapping, SPM) methods, are needed to conclusively answer this question. One recent study aimed to explore this issue by Navarro Mora and colleagues, found that there are indeed neurocircuitry changes in rats injected with pilocarpine, but they did not develop SE [34]. In other words, animals that did not experience severe seizures immediately following pilocarpine injection could still exhibit SRS approximately 8 months later, showing a long latency period resembling human pathology.

Transgenic animals are more commonly being used in PET studies, and we have conducted two studies so far. One of our studies aimed to evaluate the impact of knocking out tissue-plasminogen activator (tPA) on neuronal circuitry 
during seizures. tPA is a serine protease that converts the zymogen plasminogen (plg) to the active protease plasmin [42] and initiates a potent proteolytic cascade with a wellcharacterized function in thrombolysis. Interestingly, studies from our laboratory have shown that tPA may be a molecular mediator of seizures in animal models, and thus a potential pharmacotherapeutic target, therefore we studied seizure propagation in wild-type versus $\mathrm{PAA}^{-/-}$mice using ${ }^{18} \mathrm{FDG}-$ PET. Our results were consistent with previous studies, showing that IPA was necessary for facilitating pilocarpineinduced seizures: the seizure symptoms in the knockout animals did not exceed partial body clonus (stage 3) [2]. We conducted both ROI (regional) and SPM (voxel-based) analyses to evaluate potential effects on metabolic activity between the two genotypes. Although pilocarpine injection did significantly increase activity in the limbic system and several additional brain areas, we did not observe significant differences between genotypes with the ROI method, except in the olfactory bulb, which was active in the knockout animals. Therefore we employed a more sensitive voxel-based analysis method, which created a visual map of brain areas significantly increased in conjunction with seizure activity (via a covariate analysis). This analysis revealed clusters of activation in the hippocampus, basal forebrain and septum, and thalamus that were more widespread in wild-type mice compared to the knockout animals, consistent with the behavioral data. Interestingly, midbrain, cerebellum, and olfactory bulb increases were also unique to knockout mice. Overall, these data suggested the lack of seizure spreading mechanisms instead of reduced excitatory potential in the absence of tPA.

Secondly, we explored whether changes in glial function were a component of metabolic changes in mice following pilocarpine-induced seizures [30]. We hypothesized that such changes could parallel metabolic abnormalities observed in epileptic patients $[43,44]$. We observed differences in seizure severity when unilaterally ablating microglia in the hippocampus. Our goal using PET was also to find the brain regions involved in this behavioral divergence. We found increased ${ }^{18} \mathrm{FDG}$ uptake during seizures in the septum, thalamus, hippocampus, midbrain, and cerebellum, along with decreases in the striatum which were consistent with our previous findings [2]. However, we did see reduced metabolic activation in the ipsilateral hippocampus corresponding to the location of microglia ablation. While we interpreted this result with caution, it is possible that activated microglia may contribute a small proportion of the metabolic signal.

\section{Beyond Metabolic Imaging: Histology, MRI, and Radioligands}

An important factor in interpreting small animal imaging data is the relatively low spatial resolution compared to more traditional imaging methods, such as microscopy. However, when combined with histological methods the potential amount of information that we can derive is increased. Interestingly, one study has already explored the issue of the hypometabolic hippocampus and degree of hippocampal sclerosis [45]. Hippocampal damage was quantified with quantitative magnetic resonance imaging (QMRI) and samples of human tissue, epileptic foci removed via surgical resection, were analyzed histopathologically (neuronal loss, proliferation of glial cells, and mossy fiber sprouting). The severity of metabolic hypometabolism did not seem to correlate with hippocampal damage in these patients, thus suggesting that there may not be a direct correlation between hippocampal sclerosis and FDG imaging data. However in another study, FDG hypometabolism was significantly correlated with degree of hippocampal atrophy [12].

Future studies will continue to explore the neurocircuitry of epileptogenesis by assessing different neurotransmitter systems with radiotracer ligands to various receptors and molecular markers. Animal models are particularly suited for studying potentially newly developed radiotracer ligands that may be markers for epileptogenesis before they are explored in human patients. Several well-established radiotracers have already been examined in both human and animal models. These include the 5HT1A serotonin receptor ligand ${ }^{18} \mathrm{~F}$-FCWAY [10], dopamine D2 and D3 receptor ligand ${ }^{18} \mathrm{~F}$-Fallypride $[12,32]$, and $\mathrm{GABA}(\mathrm{A})$ density with ${ }^{11} \mathrm{C}$ Flumazenil [33]. Liew et al. found that ${ }^{18} \mathrm{~F}$-FCWAY binding was reduced in the mesial temporal region of epileptic patients despite normal MRI. They suggested that ${ }^{18} \mathrm{~F}$ FCWAY may be a more accurate measure than FDG, since FDG could not distinguish mesial from lateral temporal foci in their small patient sample [10]. Yakushev et al. showed that D2/3 receptor availability appeared to be decreased by $27 \%$ bilaterally in the anterior striatum of pilocarpinetreated rats, a finding that could be interpreted as an increase in dopamine in the synapse [32]. This result may explain why we also observed a decrease in metabolic activity in the same region of pilocarpine-treated mice [30] and may suggest that increased dopamine tonus is activated at the stage of generalization of limbic seizures to exert an inhibitory restraint on seizure propagation [32]. Werhahn and colleagues also showed reduced ${ }^{18} \mathrm{~F}$-Fallypride in the borders of epileptic foci in patients with mesial TLE and hippocampal sclerosis, warranting further clinical evaluation [12]. The study by Liefaard et al. reported that GABA(A) receptor density was decreased by $36 \%$ in the cortex of kindled rats. They suggest that this decrease is a potential mechanism underlying pharmacoresistance to midazolam [33]. Overall, these studies provide an interesting framework for future experimentation.

Although only a few studies have explored various therapeutic strategies for epilepsy in small animals thus far $[11,35,46]$, experiments toward this end are likely to increase in the near future. As suggested above by the Liefaard study, examining the receptors targeted by drugs can be an interesting method to explore therapeutic effects and pharmacoresistance. The cannabinoid system was also studied using ${ }^{18} \mathrm{~F}-\mathrm{MK}-9470$ to examine the effect of chronic treatment with valproate (VPA) and levetiracetam (LEV) [11]. This study showed that VPA, but not LEV, globally increased cerebral binding of the radiotracer suggesting an indirect effect for VPA leading to the upregulation of $\mathrm{CB} 1$ receptors and a potential new mechanism for its efficacy. Vagus nerve stimulation was also explored using 
glucose metabolism, which revealed decreased activity in the hippocampus with increases bilaterally in the olfactory bulbs and disruption in striatal activity [46]. Interestingly novel applications of deep brain stimulation (DBS) to different areas for various neurological and mental disorders are being evaluated both clinically and preclinically. Small animal imaging also provides an avenue to explore neurocircuitry changes throughout the brain as a result of DBS, as shown by Gao and colleagues. DBS to the anterior thalamic nucleus (ATN) and lesion to the ATN were evaluated in this study showing these strategies may improve symptoms by different methods. This target may be clinically relevant for epilepsy based on its connectivity to the cortex and limbic structures. ATN-DBS in naïve rats resulted in elevated metabolic activity in the target region, in the hippocampus and thalamus, and decreased activity in the frontal cortex and cingulated gyrus, while ATN-lesion only reduced activity in the ATN [35]. Importantly, metabolic imaging showed that the DBS changes were both excitatory and inhibitory and also reversible, unlike those of lesion therapies, and suggested that the hippocampus and thalamus may be engaged during DBS to ameliorate seizure spreading.

In addition to PET, functional magnetic resonance imaging (fMRI) can be useful for measuring neuronal activity changes during epileptogenesis. This imaging modality indirectly measures neuronal activity based on the hemodynamic response, the fact that more neuronal activity requires more oxygen to be delivered through the blood stream. The signal measured is referred to as the BOLD signal (meaning BloodOxygen-Level Dependence), where changes in the ratio of paramagnetic deoxyhemoglobin and diamagnetic oxyhemoglobin are measured with the MRI scanner. A review by Blumenfeld elegantly describes these methods and how they can be applied to epilepsy research [47], and a study by the same author and Engolt et al., showed interesting results [48]. The study explored the differences in brain waves (via electroencephalography (EEG)) and blood flow (fMRI) between behaviorally mild partial seizures and those which propagate and become generalized convulsive seizures. The authors found that mild seizures increased the BOLD signal (and flow related cerebral blood volume, CBV, after injection of an exogenous paramagnetic contrast) in the hippocampus, thalamus, and septum, while the signal decreased in the orbitofrontal, anterior cingulate, and retrosplenial cortex, corresponding to slow waves in the frontal cortex. However during propagated seizures, the signal to all of these regions was increased and included other areas of the primary somatosensory cortex, corresponding with fast polyspike activity. This study suggests a mechanism underlying the cognitive impairments experienced by patients exhibiting mild partial seizures, and using several imaging methods, it begins to unravel neurocircuitry patterns at the various stages of epilepsy.

\section{Summary}

The studies reviewed here, both from other researchers and including our own, show that despite some limitations, small animal imaging is an extremely useful technique for understanding circuitry changes in the brain during epileptogenesis. Future studies will continue to investigate the metabolic effects of various therapeutic modalities in animal models. As well, targeted cellular changes during seizure symptoms and treatments will continue to be explored through more specific radioligands. Furthermore, correlating circuitry changes with end point histology in animal models will expand our understanding of how cellular changes relate to global metabolic dysfunction in epileptic patients.

\section{Acknowledgments}

This work was supported by the National Institutes of Health (NIH) with funding to S. E. T. (R01NS042168). The authors are grateful to Joanna Fowler and David Alexoff for helpful discussions and Nora Ruth for editing. We would also like to thank Fritz Henn for support to M. M. M while writing this manuscript.

\section{References}

[1] A. T. Berg, S. F. Berkovic, M. J. Brodie et al., "Revised terminology and concepts for organization of seizures and epilepsies: report of the ILAE commission on classification and terminology, 2005-2009," Epilepsia, vol. 51, no. 4, pp. 676-685, 2010.

[2] M. M. Mirrione, W. K. Schiffer, J. S. Fowler, D. L. Alexoff, S. L. Dewey, and S. E. Tsirka, "A novel approach for imaging brainbehavior relationships in mice reveals unexpected metabolic patterns during seizures in the absence of tissue plasminogen activator," NeuroImage, vol. 38, no. 1, pp. 34-42, 2007.

[3] M. M. Mirrione, W. K. Schiffer, M. Siddiq, S. L. Dewey, and S. E. Tsirka, "PET imaging of glucose metabolism in a mouse model of temporal lobe epilepsy," Synapse, vol. 59, no. 2, pp. 119-121, 2006.

[4] H. I. Kornblum, D. M. Araujo, A. J. Annala, K. J. Tatsukawa, M. E. Phelps, and S. R. Cherry, "In vivo imaging of neuronal activation and plasticity in the rat brain by high resolution positron emission tomography (microPET)," Nature Biotechnology, vol. 18, no. 6, pp. 655-660, 2000.

[5] K. Goffin, W. V. Paesschen, P. Dupont, and K. V. Laere, "Longitudinal microPET imaging of brain glucose metabolism in rat lithium-pilocarpine model of epilepsy," Experimental Neurology, vol. 217, no. 1, pp. 205-209, 2009.

[6] T. J. O'Brien and B. Jupp, "In-vivo imaging with small animal FDG-PET: a tool to unlock the secrets of epileptogenesis?" Experimental Neurology, vol. 220, no. 1, pp. 1-4, 2009.

[7] Y. Guo, F. Gao, S. Wang et al., "In vivo mapping of temporospatial changes in glucose utilization in rat brain during epileptogenesis: an 18F-fluorodeoxyglucose-small animal positron emission tomography study," Neuroscience, vol. 162, no. 4, pp. 972-979, 2009.

[8] M. M. Mirrione, D. K. Konomos, I. Gravanis et al., "Microglial ablation and lipopolysaccharide preconditioning affects pilocarpine-induced seizures in mice," Neurobiology of Disease, vol. 39, no. 1, pp. 85-97, 2010.

[9] E. Assem-Hilger et al., "Central serotonin 1A receptor binding in temporal lobe epilepsy: a [carbonyl-(11)C]WAY-100635 PET study," Epilepsy and Behavior, vol. 19, no. 3, pp. 467-473, 2010. 
[10] C. J. Liew et al., " ${ }^{18}$ F-FCWAY and ${ }^{18}$ F-FDG PET in MRInegative temporal lobe epilepsy," Epilepsia, vol. 50, no. 2, pp. 234-239, 2009.

[11] K. Goffin, G. Bormans, C. Casteels et al., "An in vivo [18F]MK9470 microPET study of type 1 cannabinoid receptor binding in Wistar rats after chronic administration of valproate and levetiracetam," Neuropharmacology, vol. 54, no. 7, pp. 1103 $1106,2008$.

[12] K. J. Werhahn, C. Landvogt, S. Klimpe et al., "Decreased dopamine D2/D3-receptor binding in temporal lobe epilepsy: an [18F]Fallypride PET study," Epilepsia, vol. 47, no. 8, pp. 1392-1396, 2006.

[13] R. L. Bailey et al., "Dietary supplement use in the United States, 2003-2006," Journal of Nutrition, vol. 141, no. 2, pp. 261-266, 2011.

[14] A. Joosse et al., "Gender differences in melanoma survival: female patients have a decreased risk of metastasis," Journal of Investigative Dermatology, vol. 131, no. 3, pp. 719-726, 2011.

[15] C. Y. Fong and A. V. Delgado-Escueta, "Ictal PET in temporal lobe epilepsy," Journal of Neurology, Neurosurgery and Psychiatry, vol. 67, no. 3, p. 409, 1999.

[16] N. J. Kazemi, G. A. Worrell, S. M. Stead et al., "Ictal SPECT statistical parametric mapping in temporal lobe epilepsy surgery," Neurology, vol. 74, no. 1, pp. 70-76, 2010.

[17] J. S. Fowler and A. P. Wolf, "2-deoxy-2-[18F]fluoro-d-glucose for metabolic studies: current status," International Journal of Radiation Applications and Instrumentation Part A, vol. 37, no. 8, pp. 663-668, 1986.

[18] D. L. Alexoff, P. Vaska, D. Marsteller et al., "Reproducibility of $11 \mathrm{C}$-raclopride binding in the rat brain measured with the microPET R4: effects of scatter correction and tracer specific activity," Journal of Nuclear Medicine, vol. 44, no. 5, pp. 815$822,2003$.

[19] L. A. Shepp and Y. Vardi, "Maximum likelihood reconstruction for emission tomography," IEEE Transactions on Medical Imaging, vol. 1, no. 2, pp. 113-122, 1982.

[20] J. Qi and R. M. Leahy, "Iterative reconstruction techniques in emission computed tomography," Physics in Medicine and Biology, vol. 51, no. 15, pp. R541-R578, 2006.

[21] H. M. Hudson and R. S. Larkin, "Accelerated image reconstruction using ordered subsets of projection data," IEEE Transactions on Medical Imaging, vol. 13, no. 4, pp. 601-609, 1994.

[22] J. Nuyts, C. Michel, and P. Dupont, "Maximum-likelihood expectation-maximization reconstruction of sinograms with arbitrary noise distribution using NEC-transformations," IEEE Transactions on Medical Imaging, vol. 20, no. 5, pp. 365375, 2001.

[23] B. Bai, Q. Li, C. H. Holdsworth et al., "Model-based normalization for iterative 3D PET image reconstruction," Physics in Medicine and Biology, vol. 47, no. 15, pp. 2773-2784, 2002.

[24] C. Knoess, S. Siegel, A. Smith et al., "Performance evaluation of the microPET R4 PET scanner for rodents," European Journal of Nuclear Medicine and Molecular Imaging, vol. 30, no. 5, pp. 737-747, 2003.

[25] Y. C. Tai, A. Ruangma, D. Rowland et al., "Performance evaluation of the microPET focus: a third-generation microPET scanner dedicated to animal imaging," Journal of Nuclear Medicine, vol. 46, no. 3, pp. 455-463, 2005.

[26] G. Tarantola, F. Zito, and P. Gerundini, "PET instrumentation and reconstruction algorithms in whole-body applications," Journal of Nuclear Medicine, vol. 44, no. 5, pp. 756-769, 2003.

[27] M. E. Phelps, S. C. Huang, and E. J. Hoffman, “Tomographic measurement of local cerebral glucose metabolic rate in humans with (F-18)2-fluoro-2-deoxy-D-glucose: validation of method," Annals of Neurology, vol. 6, no. 5, pp. 371-388, 1979.

[28] E. Millan, B. Abou-Khalil, D. Delbeke, and P. Konrad, "Frontal localization of absence seizures demonstrated by ictal positron emission tomography," Epilepsy and Behavior, vol. 2, no. 1, pp. 54-60, 2001.

[29] K. Goffin, S. Dedeurwaerdere, K. Van Laere, and W. Van Paesschen, "Neuronuclear assessment of patients with epilepsy," Seminars in Nuclear Medicine, vol. 38, no. 4, pp. 227-239, 2008.

[30] G. P. Nobre et al., "Coupled-channel calculation of nonelastic cross sections using a density-functional structure model," Physical Review Letters, vol. 105, no. 20, Article ID 202502, 2010.

[31] G. Sroczynski et al., "Decision-analytic modeling to evaluate the long-term effectiveness and cost-effectiveness of HPVDNA testing in primary cervical cancer screening in Germany," GMS Health Technology Assessment, vol. 6, article Doc05, 2010.

[32] I. Y. Yakushev, E. Dupont, H. G. Buchholz et al., "In vivo imaging of dopamine receptors in a model of temporal lobe epilepsy," Epilepsia, vol. 51, no. 3, pp. 415-422, 2010.

[33] L. C. Liefaard, B. A. Ploeger, C. F. M. Molthoff et al., "Changes in GABAA receptor properties in amygdala kindled animals: in vivo studies using [11C]flumazenil and positron emission tomography," Epilepsia, vol. 50, no. 1, pp. 88-98, 2009.

[34] G. Navarro Mora, P. Bramanti, F. Osculati et al., "Does pilocarpine-induced epilepsy in adult rats require status epilepticus?" PloS one, vol. 4, article e5759, no. 6, 2009.

[35] F. Gao, Y. Guo, H. Zhang et al., "Anterior thalamic nucleus stimulation modulates regional cerebral metabolism: an FDGMicroPET study in rats," Neurobiology of Disease, vol. 34, no. 3, pp. 477-483, 2009.

[36] B. Jupp, J. P. Williams, Y. A. Tesiram, M. Vosmansky, and T. J. O'Brien, "MRI compatible electrodes for the induction of amygdala kindling in rats," Journal of Neuroscience Methods, vol. 155, no. 1, pp. 72-76, 2006.

[37] B. Jupp, J. P. Williams, Y. A. Tesiram, M. Vosmansky, and T. J. O'Brien, "Hippocampal T2 signal change during amygdala kindling epileptogenesis," Epilepsia, vol. 47, no. 1, pp. 41-46, 2006.

[38] S. Dedeurwaerdere, B. Jupp, and T. J. O’Brien, "Positron emission tomography in basic epilepsy research: a view of the epileptic brain," Epilepsia, vol. 48, supplement 4, pp. 56-64, 2007.

[39] J. Duncan, "The current status of neuroimaging for epilepsy," Current Opinion in Neurology, vol. 22, no. 2, pp. 179-184, 2009.

[40] J. S. Duncan, "Neuroimaging methods to evaluate the etiology and consequences of epilepsy," Epilepsy Research, vol. 50, no. 1-2, pp. 131-140, 2002.

[41] B. Jupp and T. J. O’Brien, "Application of coregistration for imaging of animal models of epilepsy," Epilepsia, vol. 48, supplement 4, pp. 82-89, 2007.

[42] J. D. Vassalli, A. P. Sappino, and D. Belin, "The plasminogen activator/plasmin system," Journal of Clinical Investigation, vol. 88, no. 4, pp. 1067-1072, 1991.

[43] E. E. Altay, A. J. Fessler, M. Gallagher et al., "Correlation of severity of FDG-PET hypometabolism and interictal regional delta slowing in temporal lobe epilepsy," Epilepsia, vol. 46, no. 4, pp. 573-576, 2005.

[44] S. Vielhaber, J. H. Von Oertzen, A. F. Kudin et al., "Correlation of hippocampal glucose oxidation capacity and interictal FDG-PET in temporal lobe epilepsy," Epilepsia, vol. 44, no. 2, pp. 193-199, 2003. 
[45] S. Lamusuo, L. Jutila, A. Ylinen et al., "[18F]FDG-PET reveals temporal hypometabolism in patients with temporal lobe epilepsy even when quantitative MRI and histopathological analysis show only mild hippocampal damage," Archives of Neurology, vol. 58, no. 6, pp. 933-939, 2001.

[46] S. Dedeurwaerdere, B. Cornelissen, K. Van Laere et al., "Small animal positron emission tomography during vagus nerve stimulation in rats: a pilot study," Epilepsy Research, vol. 67, no. 3, pp. 133-141, 2005.

[47] H. Blumenfeld, "Functional MRI studies of animal models in epilepsy," Epilepsia, vol. 48, supplement 4, pp. 18-26, 2007.

[48] D. J. Englot, A. M. Mishra, P. K. Mansuripur, P. Herman, F. Hyder, and H. Blumenfeld, "Remote effects of focal hippocampal seizures on the rat neocortex," Journal of Neuroscience, vol. 28, no. 36, pp. 9066-9081, 2008. 


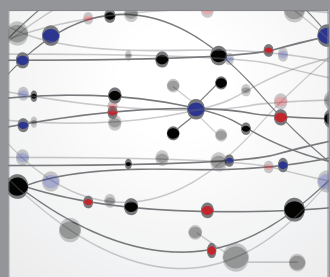

The Scientific World Journal
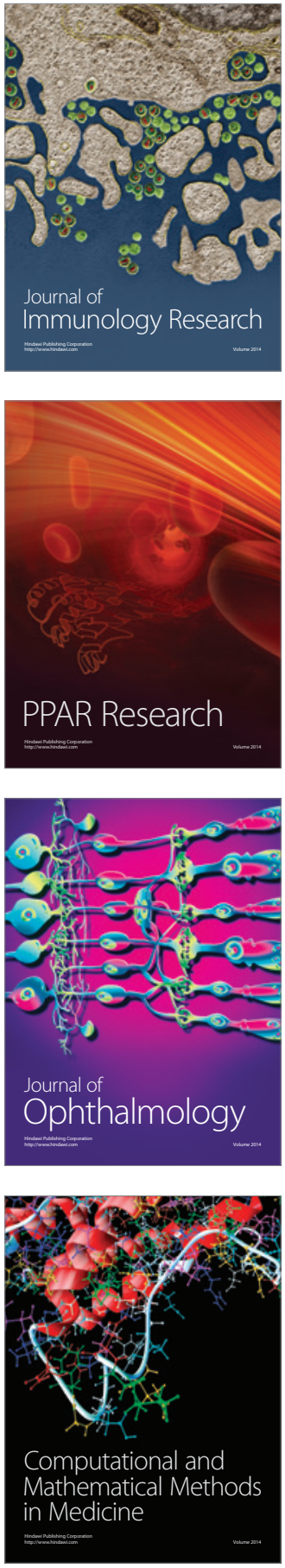

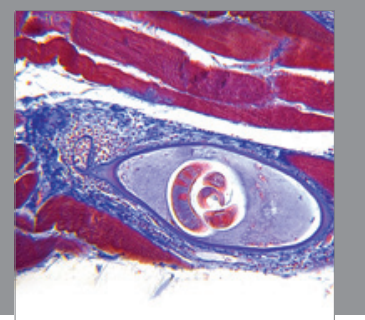

Gastroenterology

Research and Practice
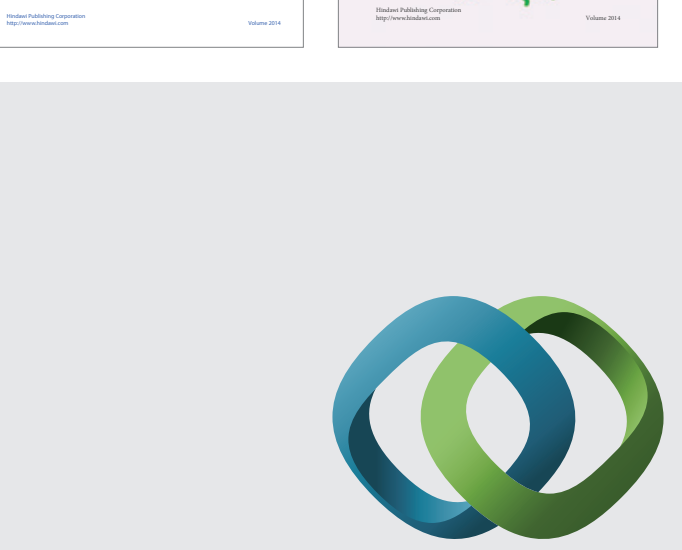

\section{Hindawi}

Submit your manuscripts at

http://www.hindawi.com
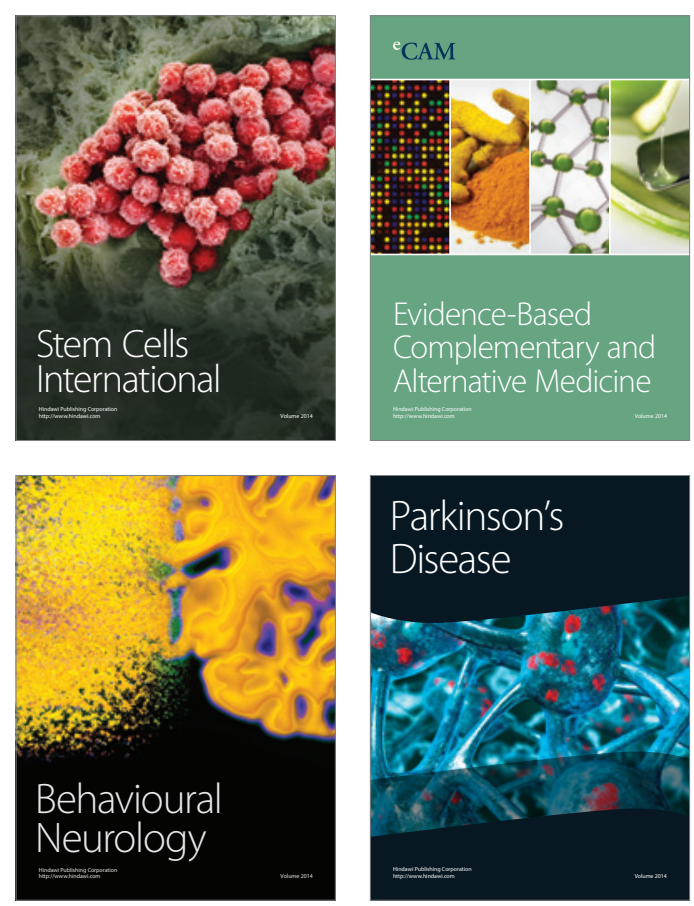

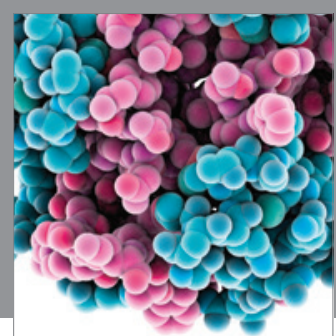

Journal of
Diabetes Research

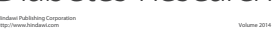

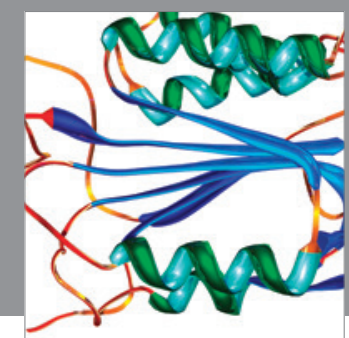

Disease Markers
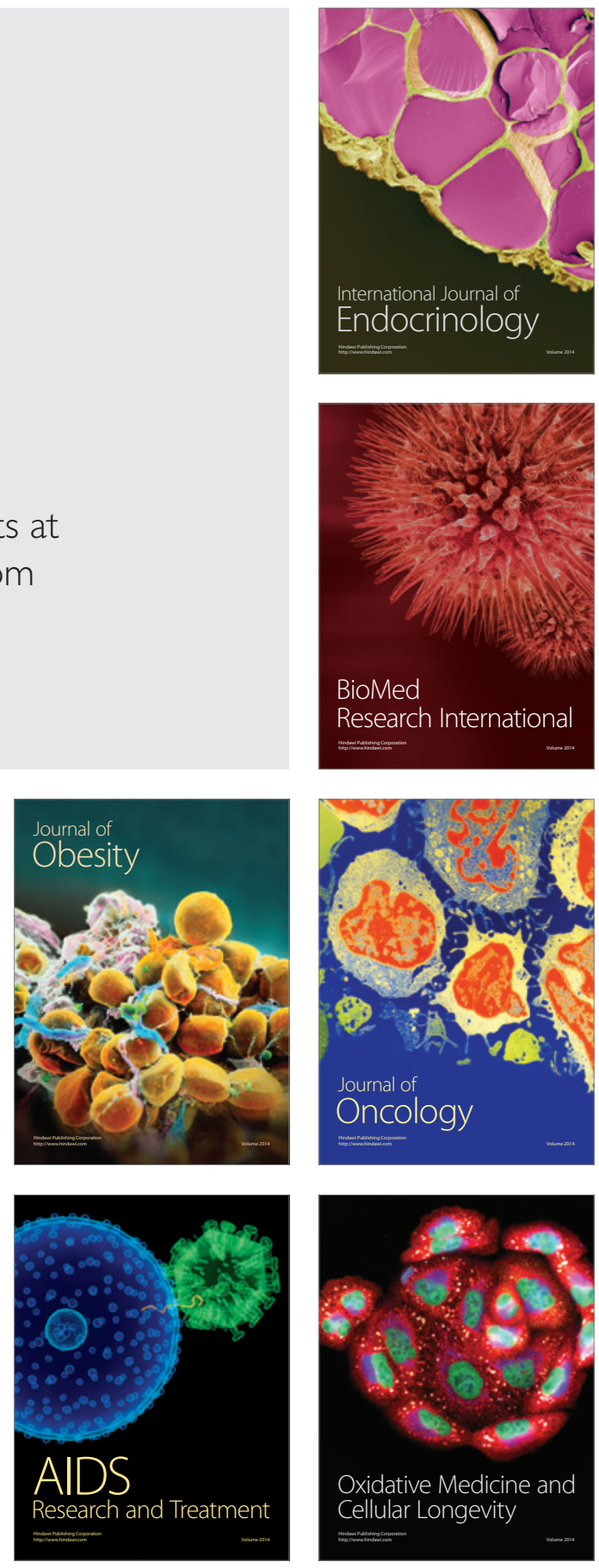